\title{
Personalização do E-Learning Baseado no Nível de Aquisição de Conhecimentos do Aprendiz
}

\author{
Eduardo Henrique Gomes ${ }^{1,2}$ \\ Orientador: Prof. Dr. Edson P. Pimentel ${ }^{1}$ \\ ${ }^{1}$ Universidade Federal do ABC (UFABC) \\ Santo André - SP - Brasil \\ ${ }^{2}$ Instituto Federal de Educação Ciência e Tecnologia de São Paulo (IFSP) \\ Cubatão - SP - Brazil \\ \{eduardo.gomes, edson.pimentel\}@ufabc.edu.br
}

\begin{abstract}
The learner's Knowledge Acquisition Level (KAL) can be used to generate personalized learning plans. However, the personalization of education mediated by Learning Managment Systems (LMS) has been little used due the lack of interoperability of these environments with Adaptive Hypermedia Systems (AHS), which generally include authoring tool and adaptation mechanism. This gap can be supplied with the framework GALE that provides adaptation functionalities beyond the feature of integration with existing LMS. In this context, this work aims to model and create adaptive units, based on KAL of the learner, through GALE mechanism. This goal will be achieved by the development of an adaptive unit (course encompassing modeling contents, rules creation, selection of instructional material and its experimentation using the mechanism GALE. It is intended to show the effectiveness of the process by analysing adaptation to established stereotypes (basic, intermediate, advanced) based on KAL.
\end{abstract}

Resumo. O Nível de Aquisição de Conhecimentos (NAC) do estudante pode ser utilizado para a geração de planos de aprendizado personalizado. No entanto, a personalização da educação mediada pelos Ambientes Virtuais de Aprendizagem (AVA) tem sido pouco utilizada devido a falta de interoperabilidade desses ambientes com os Sistemas de Hipermídia Adaptativa (SHA), que geralmente englobam ferramenta de autoria e mecanismo de adaptação. Essa lacuna pode ser suprida com o framework GALE que fornece funcionalidades de adaptação além de da possibilidade de integração com AVAs existentes. Nesse contexto, este trabalho tem por objetivo geral modelar e criar unidades adaptativas, baseadas no NAC do aprendiz, por meio do mecanismo GALE. Por meio da elaboracão de uma unidade adaptativa (curso), englobando modelagem de conteúdos, criacão de regras, seleção de material instrucional, e sua experimentacão utilizando o mecanismo GALE pretende-se mostrar a eficácia do processo analisando-se a adaptacão aos estereótipos estabelecidos (básico, intermediário, avançado) baseados no NAC.

\section{Introdução}

A disseminação no uso de ambientes E-learning amplia também a possibilidade de se empregar a personalização da educação, ou seja, o tratamento mais individualizado das ne- 
cessidades de aprendizagem uma vez que esses ambientes são capazes de registrar dados da interação do estudante, suas realizações e consequentemente suas lacunas. No entanto, na prática esta personalização tem sido pouco disseminada possivelmente pela carência de ferramentas de autoria para a produção de conteúdos adaptativos que facilitem a criação de critérios de adaptação pelos próprios educadores.

Para que seja possível implementar um modelo de aprendizagem flexível e inteligente, que privilegie o perfil individual de cada aluno, levando em consideração suas características e preferências, os AVAs devem ser capazes de fornecer ou integrar-se a Sistemas de Hipermídia Adaptativa (SHA).

Perante isto, as seguintes questões aparecem na tentativa de se identificar os motivos da não utilização da personalização nos ambientes de E-learning, tais como:

1. Existem ambientes de aprendizagem adaptativa (framework), que consigam se integrar aos AVAs existentes e já massificados?

2. Havendo esses ambientes de aprendizagem adaptativa integráveis a AVAS populares, as ferramentas de autoria de adaptatividade são amigáveis e de fácil uso no que diz respeito à estruturação da base de conhecimentos, criação de regras, etc?

Nesse contexto, o framework adaptativo GALE do Projeto GRAPPLE, apareceu como uma opção para prover funcionalidades de adaptação de maneira intercambiável entre os AVAs existentes, além de disponibilizar a ferramenta de autoria visual GAT.

Nesse sentido este trabalho tem por objetivo modelar e criar unidades adaptativas baseadas no nível de aquisição de conhecimentos do aprendiz. Apresenta-se ainda uma experimentação por meio do mecanismo GALE.

Este estudo está organizado com a seguinte estrutura: o Capítulo 2 apresenta os conceitos sobre a personalização do ensino e os tipos de adaptação no E-learning. O Capítulo 3 trata dos Sistemas de Hipermídia Adaptativa. O Capítulo 4 aborda o processo de autoria de uma unidade adaptativa com o uso de ferramentas externas, para implementação no servidor GALE. O Capítulo 5 apresenta as considerações finais.

\section{Personalização e Adaptação no E-Learning}

O modelo de sala de aula tradicional, no qual um único professor expõe conteúdos a diversos alunos, não consegue prover uma educação personalizada capaz de tratar as necessidades individuais de cada estudante. Por outro lado, a Educação mediada por tecnologias de informação através do E-learning, têm potencial para disponibilizar ao estudante o conteúdo adequado em função das suas próprias necessidades de aprendizagem [U.S.A 2010].

Segundo [Kahiigi et al. 2008] que pesquisou o estado da arte em sistemas de Elearning, a personalização da aprendizagem é definida como "uma abordagem de aprendizagem que facilita e suporta uma aprendizagem individualizada, na qual cada aluno tem um caminho de aprendizagem que atende suas necessidades e interesses, tornando esse caminho produtivo e significativo".

No âmbito da Informática na Educação, a personalização é estudada sob a ótica dos mecanismos de adaptação e também dos parâmetros de personalização. Tradicionalmente na literatura, sob o ponto de vista pedagógico, existem três tipos de adaptação: 
baseada na interface, baseada no fluxo e baseada no conteúdo. Sob o ponto de vista técnico a taxonomia de [Brusilovsky 2001] descreve os tipos de adaptação encontrados na Hipermídia Adaptativa e cujos principais tipos são: Adaptação de apresentação e Adaptação de navegação.

\subsection{Parâmetros para Adaptação de Conteúdos}

Em sua pesquisa, [Essalmi et al. 2010] destaca que os parâmetros de personalização constituem a fonte para a personalização nos ambientes de E-learning. Um parâmetro de personalização pode ser visto como um conjunto de valores. Por exemplo, o parâmetro de personalização nível de aquisição de conhecimentos do aprendiz, pode ser representado pelo conjunto (estereótipo): básico, intermediário e avançado. Já um conjunto de parâmetros de personalização é denominado como uma estratégia de personalização.

[Essalmi et al. 2010] mostra que o parâmetro de personalização "nível de conhecimento do aprendiz", é a estratégia de personalização mais utilizada hoje em dia e também a mais significativa. Na pesquisa de [O'Donnell 2012], o conhecimento prévio também foi percebido como a característica mais importante do aprendiz, para que a personalização seja alcançada mais facilmente. Isso corrobora a escolha do uso do nível de aquisição de conhecimentos pelo aprendiz neste trabalho.

Para estabelecer uma medida capaz de identificar o grau de conhecimentos que um aprendiz possui, será utilizado o estudo de [Pimentel 2006], que utiliza como métrica cognitiva, o Nível de Aquisição de Conhecimentos - (NAC) e é definido como: "uma medida que indica o grau de conhecimentos do aprendiz em um determinado conteúdo, de um domínio de conhecimento, naquele instante”.

Uma forma de se estabelecer o NAC inicial é através de uma avaliação diagnóstica, sua finalidade é identificar a existência ou não de requisitos prévios. Nesse sentido, o próprio aluno, através de questionário, precisará indicar o quanto sabe sobre todos os conceitos que serão abordados, antes de passar pelo processo instrucional.

Uma maneira de se permitir que um SHA comece rapidamente sua interação personalizada com o aprendiz é realizado com o uso de estereótipos. Um estereótipo representa uma coleção de atributos que aparecem em um grupo de pessoas. O método mais comum utilizado para representar evidências de conhecimento entre aprendizes, em diversos contextos, é o modelo do aluno que apresenta sobreposição de conhecimento dos alunos, como um subconjunto do conhecimento de domínio total [Palazzo 2002]. Ou seja, muitas vezes é possível observar padrões entre alunos, e grupos de alunos, com características semelhantes dentro de categorias [Kay 2000].

\subsection{Organização do Conhecimento para Adaptação de Conteúdos}

Várias são as técnicas de representação de conhecimento. No âmbito dos ambientes computacionais aplicados à educação, as técnicas mais utilizadas são os mapas conceituais, mapas hierárquicos e mais recentemente as ontologias [Pimentel 2006].

Desenvolvidos em 1972 por Novak na universidade de Cornell, os mapas conceituais se basearam na psicologia de aprendizagem de David Ausubel [Ausubel et al. 1968]. A ideia principal dos mapas conceituais é que a aprendizagem ocorre por assimilação de novos conceitos e proposições em conceitos existentes, essas estruturas proposicionais 
são assimiladas pelo aprendiz e também são conhecidas como estruturas cognitivas do indivíduo [Novak and Cañas 2006].

Os mapas conceituais são ferramentas gráficas para organizar e representar o conhecimento de maneira que permitam a transferência de mensagens complexas de uma forma altamente estruturada, no qual conceitos mais gerais ficam no topo do mapa, e os conceitos mais específicos são dispostos hierarquicamente abaixo. Uma das ferramentas mais utilizadas é a CmapTools ${ }^{1}$ [CmapTools 2008].

\section{Sistemas de Hipermídia Adaptativa}

Os Sistemas de Hipermídia Adaptativa (SHA) têm se mostrado muito adequados para a inserção de características de personalização da educação em Ambientes Virtuais de Aprendizagem. De acordo com [Brusilovsky et al. 1998], num SHA existem três características importantes: (a) é um sistema de hipertexto ou hipermídia, (b) armazena informações sobre o usuário e (c) adapta a aparência do sistema para o usuário.

Pesquisas em modelos de sistemas de hipermídia adaptativa, apontam para uma característica fundamental, que é a separação entre a estratégia de conteúdo e a adaptação da unidade adaptativa, isso aumenta a capacidade de reutilização em comparação com abordagens que combinam todos os fatores em um mesmo modelo [Brusilovsky et al. 1998]. Alguns exemplos de SHA conhecidos são: Interbook [Brusilovsky et al. 1998], AHA! [De Bra and Stash 2002], My Online Teacher [Cristea and de Mooij 2003] e SQL-Tutor [Mitrovic 2003].

A autoria de hipermídia adaptativa é um esforço notoriamente difícil, o desenvolvimento de uma unidade adaptativa pode ser complexo, porque o comportamento adaptativo pode ser complexo, embora seus resultados possam ser muito valiosos, gerando, por exemplo, no contexto educacional experiências personalizadas de aprendizagem. A solução deste problema conforme relata [Hendrix 2010], são ferramentas de autoria que sejam automatizadas e retirem do autor a função trabalhosa de programação.

Nesse trabalho, buscou-se explorar a plataforma de hipermidia adaptativa do projeto GRAPPLE que é detalhado a seguir.

O projeto GRAPPLE (Generic Responsive Adaptive Personalised Learning Environment) [GRAPPLE 2010] é uma abreviação para "Ambiente de Aprendizagem Genérico, Personalizado e Responsivo" é uma plataforma de hipermídia adaptativa que se integra com os principais AVAs disponíveis no mercado utilizando uma abordagem orientada a serviços.

Dentre suas principais ferramentas, destaca-se aquela responsável pela criação de conteúdos de aprendizado adaptativos, denominada GAT (Grappple Authoring Tool). Uma das principais vantagens desta proposta é que a mesma consiste em um ambiente visual, voltado para facilitar a criação de conteúdo pelos autores. Também pode-se destacar o ambiente GALE (framework de aprendizagem adaptativa) e a linguagem GAM (Gale Adaptation Model), todas estas construídas no contexto do projeto GRAPPLE.

[Hendrix 2010] descreve brevemente o modelo utilizado na ferramenta de autoria de conteúdo do projeto GRAPPLE. Tratam-se de três camadas: Modelo de Domínio,

\footnotetext{
${ }^{1} \mathrm{~A}$ ferramenta CmapTools pode ser encontrada no endereço: http://cmap.ihmc.us.
} 
Modelo de Usuário e Modelos de Adaptação, através das quais é possível criar objetos de aprendizado que ofereçam possibilidade de adaptação de conteúdo em AVAs.

\section{Unidade Adaptativa Baseada no NAC}

Para cada conceito da unidade adaptativa, um recurso de aprendizagem precisa ser escrito e fornecido através de uma URL. No GALE os tipos de conteúdo mais utilizados como recurso de aprendizagem são o XHTML e o XML. Os arquivos da unidade adaptativa contendo todos os recursos foram colocados na pasta de aplicações do servidor GALE.

O método utilizado para a definição da estratégia de personalização foi a técnica do arquivo GAM empacotado, no qual se insere no arquivo concepts.gam, de formato puramente textual e que é colocado na pasta contendo os recursos, todos os conceitos do modelo de domínio. Também são especificados neste arquivo, a hierarquia de conceitos baseando-se no mapa conceitual, os requisitos prévios de cada conceito e são declaradas todas as variáveis e funções utilizadas no processo como mostrado na Figura 1. Por padrão o GALE procura um arquivo chamado concepts.gam dentro de cada pasta e o processa.

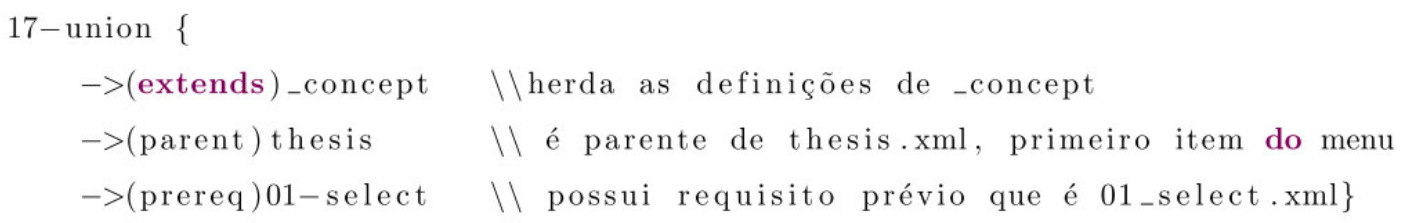

Figure 1. Fragmento de Código do Arquivo .gam - Relação Entre Conceitos.

A seguir apresenta-se uma experimentação, criando-se uma unidade adaptativa sobre a linguagem SQL, criada com ferramentas externas e que utilizaram as seguintes abordagens:

- Modelagem de domínio de conhecimento fazendo uso de mapas conceituais;

- Definição da estratégia de personalização através do parâmetro NAC;

- Configuração do perfil inicial do aprendiz a partir de avaliação diagnóstica;

- Formação de grupos de estereótipos.

- Adaptação dos recursos utilizando a técnica do arquivo GAM empacotado.

\subsection{Modelo de Domínio Utilizando Mapas Conceituais}

Nesse trabalho, para a representação do conhecimento, optou-se pela criação de um mapa conceitual $^{2}$ através da ferramenta CmapTools [CmapTools 2008], no qual foi implementado o modelo de domínio que contém todos os conceitos do domínio de conhecimento.

Como este trabalho escolheu utilizar ferramentas externas para a implementação da unidade adaptativa, o mapa conceitual serviu como um guia para a programação das regras, facilitando a visualização da hierarquia de conceitos, as relações entre conceitos e requisitos prévios existentes. Além de ser utilizado durante a introdução do módulo de aprendizagem, o mapa servirá para a ativação do conhecimento aprendido em aulas anteriores e também como um mecanismo de recuperação de informação.

\footnotetext{
${ }^{2} \mathrm{O}$ mapa de conceitos da unidade adaptativa desenvolvida, pode ser acessado através do endereço: http://cmaps.cmappers.net/rid=1L3VPSKKX-10L6Q3Y-52MRG/ehgomes.
} 


\subsection{Nível de Aquisição do Conhecimento Através do Grau de Confiança}

Nesse trabalho, a fim de se estabelecer uma medida capaz de identificar o grau de conhecimentos que o aprendiz possui, foi utilizado o NAC. Como o NAC é uma medida por conceito, e em decorrência da escolha do uso de três níveis de estereótipos, os conceitos foram organizados por blocos, ou seja, blocos de conceitos básicos (A), blocos de conceitos intermediários (B) e blocos de conceitos avançados (C), como ilustrado na Figura 2. Também colocou-se pesos por blocos: 1, 2 e 3 respectivamente, dessa forma o NAC é calculado utilizando-se a média ponderada.

Para a obtenção do NAC inicial, este trabalho desenvolveu um formulário de avaliação diagnóstica que se utilizará do grau de confiança do aprendiz. Cada pergunta do formulário está associado a um conceito como mostrado na Figura 2, o aprendiz deve assinalar valores entre 0 e 5 para indicar o seu grau de confiança nos próprios conhecimentos.

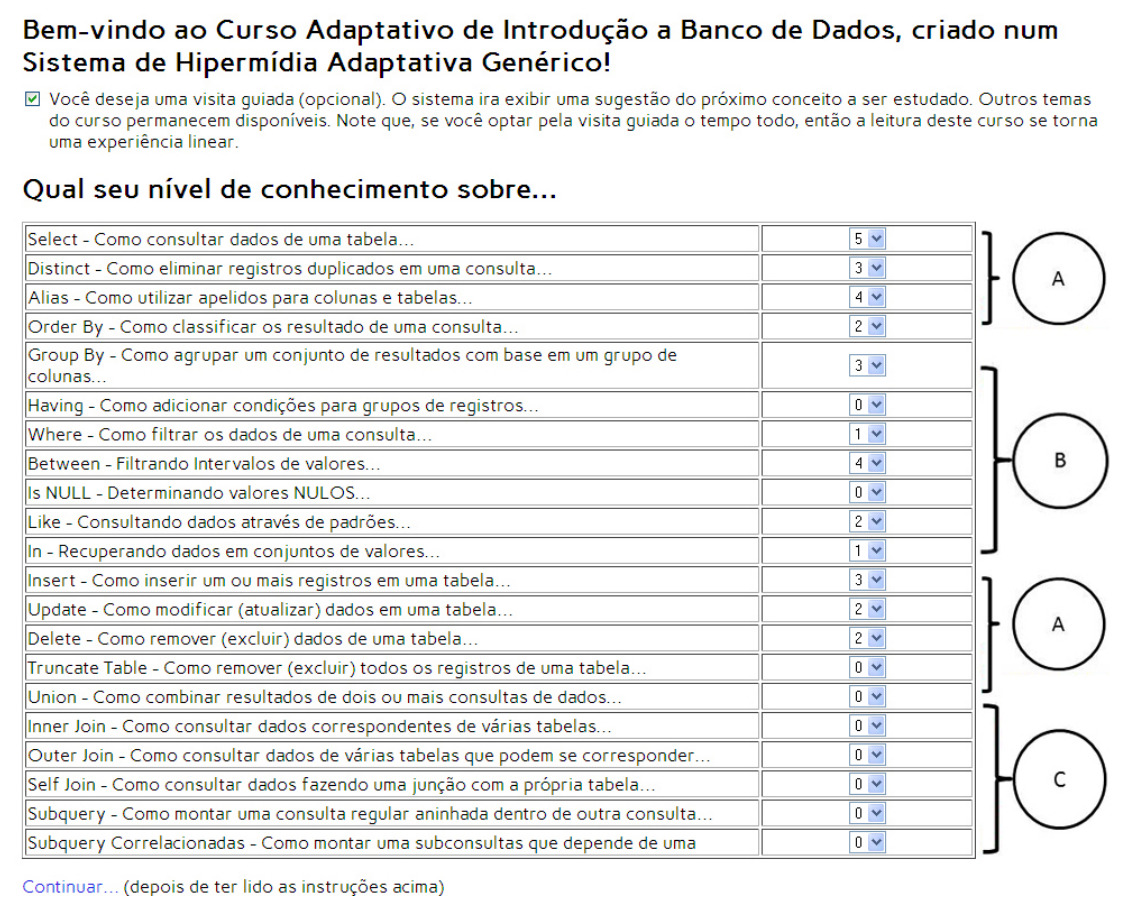

Figure 2. Tela Inicial Contendo o Formulário de Avaliação Diagnóstica.

\subsection{Modelagem do Aprendiz em Estereótipos}

Com a finalidade de construir um modelo inicial do aprendiz, que pudesse ser utilizado inicialmente logo na entrada do aprendiz no sistema, a abordagem usada foi a categorização do aprendiz em estereótipos através do NAC.

Este trabalho utiliza o parâmetro de personalização nível de conhecimento do aprendiz, de [Chorfi and Jemni 2004] que utiliza três estereótipos distintos: beginner, intermediate e expert. O aprendiz é atribuído a um dos três estereótipos, dependendo de seu desempenho no formulário de avaliação diagnóstica, ou seja, de acordo com o NAC.

\subsection{Adaptação dos Recursos da Unidade Adaptativa}

Se o aprendiz está no primeiro acesso da unidade adaptativa e já enviou o formulário, o primeiro conceito a ser mostrado é o recurso thesis.xhtml, que disponibiliza o mapa 
conceitual do domínio de conhecimento. Também mostra as instruções de navegação da unidade adaptativa, que podem ser visualizadas na Figura 3, e que se utiliza da técnica de anotação de links da taxonomia de Brusilovsky.

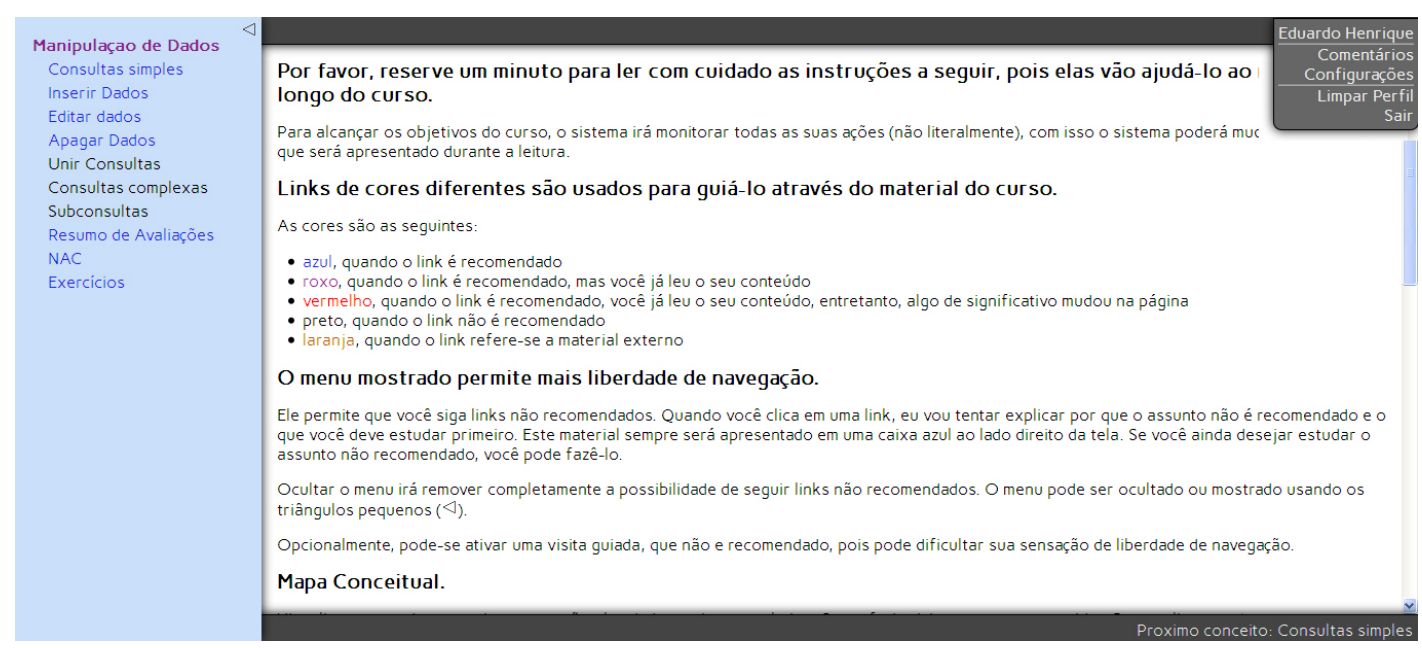

\section{Figure 3. Primeiro Conceito da Unidade Adaptativa e as Instruções de Navegação.}

Na Figura 3, pode-se visualizar a hierarquia de conceitos que formam o menu da unidade adaptativa. Este menu pode ser ocultado, porém os links não adequados (pretos) não serão mostrados dentro do texto, pois se utilizam da técnica de ocultação de links. Esta opção de ocultação do menu retira do aprendiz a possibilidade de acessar um conceito não adequado, segundo a estratégia, e não é indicada.

Pode-se ver no rodapé da Figura 3, que um conceito é sempre sugerido ao aprendiz, conceitos não adequados não serão sugeridos. O texto do formulário mostrado na Figura 2 alerta que, ao optar pela navegação guiada, a leitura do hipertexto torna-se uma experiência linear de conceitos adequados. Está técnica é chamada de orientação direta.

Tomando como exemplo o recurso Consultas Complexas, que é um conceito inadequado ao aprendiz neste momento, pois o conceito Consultas Simples, é requisito prévio. Se o aprendiz desejar ler o conceito Consultas Complexas, o arquivo concept.gam detecta que o conceito é inadequado, e chama um arquivo de nome unsuitable.xhtml, que mostra as informações como visualizado na Figura 4.

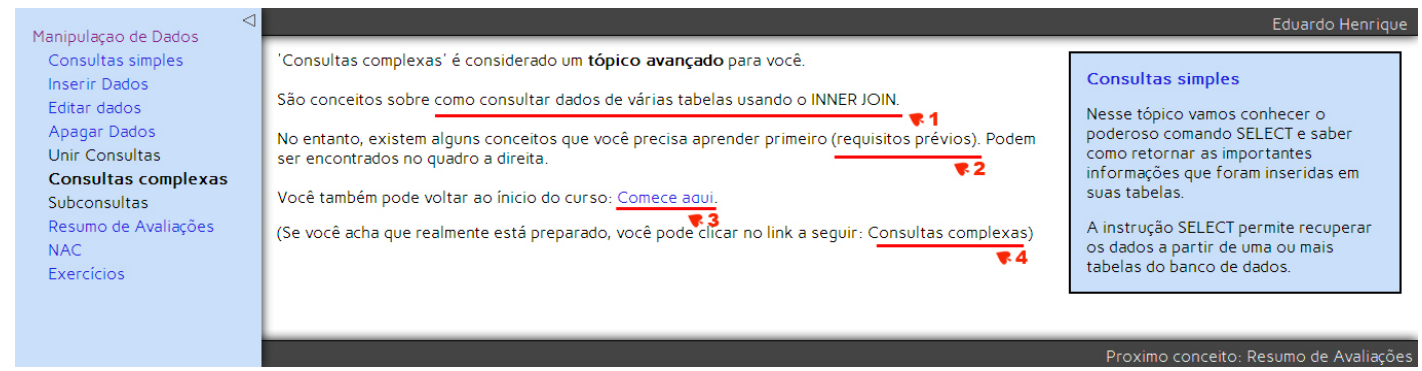

Figure 4. Adaptação em Conceito Não Adequado.

O arquivo unsuitable.xhtml, exibe o titulo do conceito, diz que é um tópico avançado e exibe uma prévia do que o aprendiz poderá aprender no conceito, como vi- 
sualizado na observação 1 da Figura 4. Também lista num div a direita da página, o titulo e o conteúdo do recurso prévio, no caso em questão: Consultas Simples. São ainda oferecidas duas opções para o aprendiz, a primeira recomenda que o aprendiz inicie pelo requisito prévio, visualizado na observação 3 da Figura 4. A segunda, permite que o aprendiz acesse o conteúdo do conceito não adequado Consultas Complexas, através do link mostrado na observação 4 da Figura 4. Note que o texto do link está na cor preta, utilizando a técnica de ocultação de links. Se o aprendiz acessar o conceito não adequado Consultas Complexas, ele tem acesso ao conteúdo. Porém, o requisito prévio, permanece no quadro a direita do recurso.

No final do conceito, é inserido o arquivo unsuitable-question.xhtml, no qual o aprendiz é lembrado mais uma vez, que este não era um conceito adequado, e pergunta se o aprendiz entendeu o conteúdo, se sim, ele pode clicar no link e este conceito não será mais inadequado, consequentemente o requisito prévio não é mais necessário. $O$ GALE também armazena que o aprendiz visitou um conceito não adequado, e disse entender seu conteúdo. Se o aprendiz visitar conceitos não adequados subsequentemente, o GALE saberá que não é a primeira vez que isso ocorre, e permite que o aprendiz não seja tratado como beginner ou intermediate. Passando assim, a não ser mais importunado com requisitos prévios e passa a ser classificado como expert.

Outra estratégia de adaptação utilizada neste estudo de caso, é a inserção de conteúdos dentro de um recurso, com base no estereótipo do aprendiz. O conteúdo inserido são três versões de arquivos .xml, que são: 01-select-beginner.xml, 01-selectintermediate.xml e 01-select-expert.xml, do conceito Consultas Simples. Pode-se visualizar na Figura 5, que o resultado final, exibe versões diferentes para cada estereótipo.

Para o usuário Beginner, o conteúdo é mais detalhado, possui figuras auto explicativas e os exemplos de resultados dos comandos de exemplo. Para o usuário Intermediate, foram removidas as figuras auto explicativas. E para o usuário Expert, ficaram apenas o organizador prévio (problema) e a sintaxe do comando.

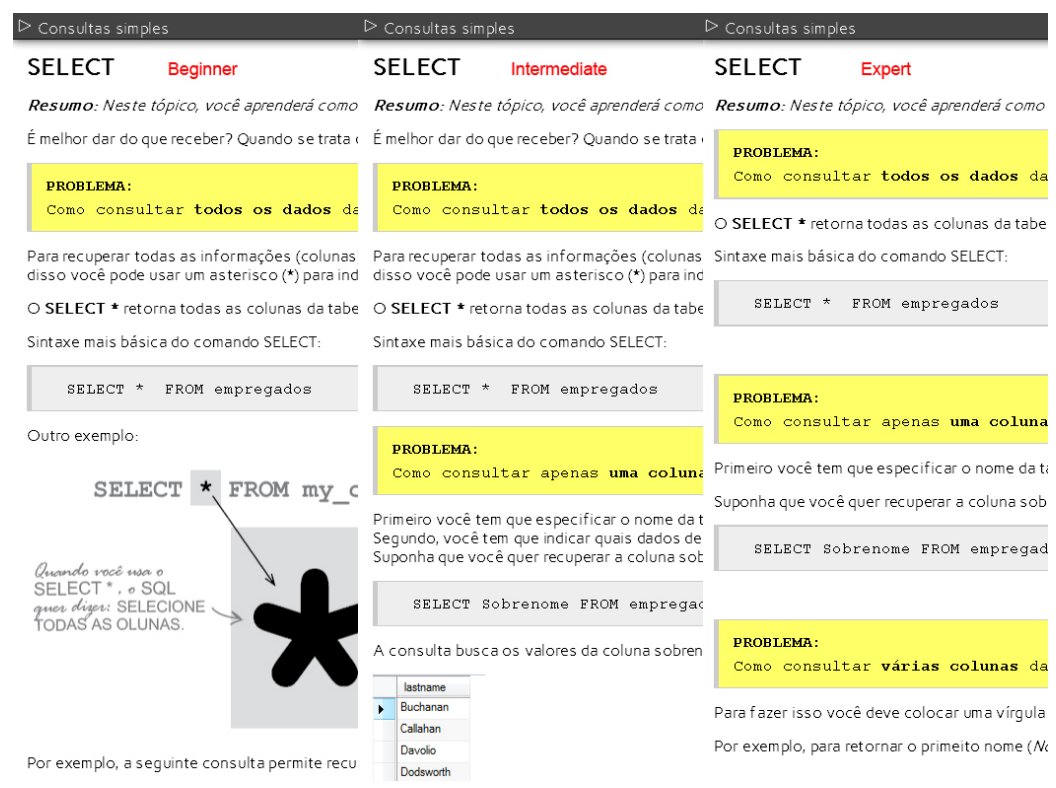

Figure 5. Versões do Recurso Consulta Simples Adaptado aos Estereótipos. 
Uma outra técnica que foi utilizada, é a inserção de fragmentos dentro de um recurso. Esta técnica, tem a vantagem de armazenar as ações do aprendiz, para que sob determinada condição, os fragmentos sejam inseridos e o aprendiz alertado que o conceito já visitado sofreu alteração significativa, e deve ser revisitado (link vermelho). A Figura 6, ilustra esse comportamento. Aprendiz visitou o conceito Editar Dados, o conceito Inserir Dados, e o conceito Consulta Simples e todos seus filhos. Após isso, foi alertado que o conceito Editar Dados sofreu alteração.

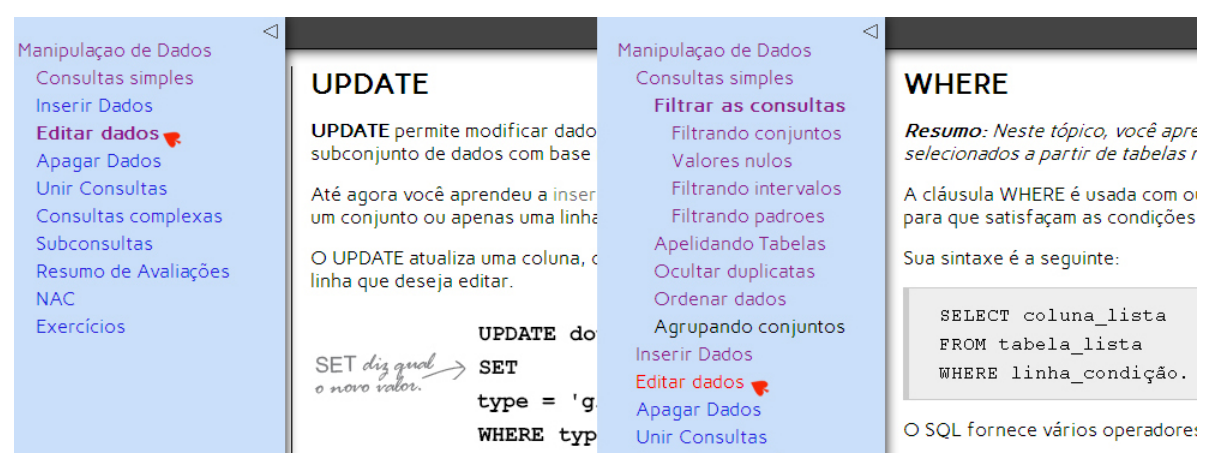

Figure 6. Conceito Editar Dados Sofrendo Alteração e Alertando Aprendiz.

O fragmento inserido no conceito Editar Dados, são variações do comando UPDATE, que possuem maior complexidade e exigem o conhecimento dos "filhos" do conceito Consulta Simples e do conceito Inserir Dados. Os conceitos inseridos são: Operações matemáticas no UPDATE e UPDATE com a expressão CASE.

\section{Considerações Finais e Trabalhos Futuros}

As observações do experimento deste trabalho indicam um resultado bastante relevante da incorporação no modelo de usuário, do Nível de Aquisição de Conhecimentos do aprendiz (NAC). Isso possibilitou a categorização dos aprendizes em estereótipos, possibilitando que o GALE inicia-se sua interação personalizada. Esses fatores evidenciaram, que o modelo de usuário e o modelo de adaptação do GALE é genericamente flexível. Também, constatou-se a eficiência da utilização dos mapas conceituais, para organizar e representar o conhecimento, facilitando assim o desenvolvimento das regras nas unidades adaptativas.

Outra contribuição deste trabalho, é a de que a tecnologia descrita nesta dissertação, pode servir como uma diretriz para que um autor desenvolva uma unidade adaptativa, uma vez que cumpre todas as etapas, desde a modelagem de conteúdos, criação de regras, seleção de material instrucional, e verificação do grau de adaptatividade, de modo que possa servir como um tutorial para futuras iniciativas.

A fim de tentar corroborar a expectativa de que a personalização produz ganhos no processo de ensino aprendizagem, considera-se como trabalho futuro a realização do estudo de caso avaliação da personalização do E-learning, a fim de provar sua utilidade em termos de eficácia educacional.

\section{References}

Ausubel, D. P., Novak, J. D., Hanesian, H., et al. (1968). Educational psychology: A cognitive view. 
Brusilovsky, P. (2001). Adaptive hypermedia. User Model. User-Adapt. Interact., 11(12):87-110.

Brusilovsky, P., Eklund, J., and Schwarz, E. (1998). Web-based education for all: a tool for development adaptive courseware. Computer Networks and ISDN Systems.

Chorfi, H. and Jemni, M. (2004). Perso: Towards an adaptive e-learning system. Journal of Interactive Learning Research, 15(4):433-447.

CmapTools (2008). Cmap tools knowledge modeling kit.

Cristea, A. I. and de Mooij, A. (2003). Adaptive course authoring: My online teacher. In Telecommunications, 2003. ICT 2003. 10th International Conference on. IEEE.

De Bra, P. D. and Stash, N. (2002). Aha! adaptive hypermedia for all.

Essalmi, F., Ayed, L. J. B., Jemni, M., Graf, S., et al. (2010). A fully personalization strategy of e-learning scenarios. Computers in Human Behavior, 26(4):581-591.

GRAPPLE (2010). Generic responsive adaptive personalized learning environment project.

Hendrix, M. (2010). Supporting Authoring of Adaptive Hypermedia. PhD thesis, University of Warwick, Department of Computer Science.

Kahiigi, E. K., Ekenberg, L., Hansson, H., Tusubira, F., and Danielson, M. (2008). Exploring the e-learning state of art. EJEL, 6(2).

Kay, J. (2000). Stereotypes, student models and scrutability. In Intelligent Tutoring Systems, pages 19-30. Springer.

Mitrovic, A. (2003). An intelligent sql tutor on the web. Int. J. Artif. Intell. Ed., 13:173197.

Novak, J. D. and Cañas, A. J. (2006). The theory underlying concept maps and how to construct them. Florida Institute for Human and Machine Cognition, 1.

O’Donnell, E., S. M. W. V. . O. L. (2012). Academics' views on personalised e-learning in higher education. In ICEP 2012: International Conference on Engaging Pedagogy.

Palazzo, L. A. M. (2002). Sistemas de hipermídia adaptativa. In XXII Congresso da Sociedade Brasileira de Computação (SBC). Anais.

Pimentel, E. P. (2006). Um modelo para avaliação e acompanhamento contínuo do nível de aquisição de conhecimentos do aprendiz. $\mathrm{PhD}$ thesis, Instituto Tecnológico de Aeronáutica. São José dos Campos: ITA.

U.S.A, D. E. (2010). National Education Technology Plan 2010. Washington, DC. 